\title{
Reactor activations to constrain astrophysically relevant cross sections
}

\author{
Sophia Dellmann ${ }^{1}$, René Reifarth ${ }^{1}$, Mario Weigand ${ }^{1}$, Klaus Eberhardt ${ }^{2}$, Ruchi Garg ${ }^{3,4}$, \\ Christopher Geppert ${ }^{2}$, Tanja Heftrich ${ }^{1}$, Deniz Kurtulgil ${ }^{1}$, and Claudia Lederer-Woods ${ }^{3}$ \\ ${ }^{1}$ Goethe University, Frankfurt, Germany \\ ${ }^{2}$ Johannes Gutenberg University, Mainz, Germany \\ ${ }^{3}$ University of Edinburgh, United Kingdom \\ ${ }^{4}$ Michigan State University, USA
}

\begin{abstract}
The determination of astrophysically relevant neutron-induced cross sections is particularly difficult when the involved isotopes are radioactive or the cross sections are very small. Activation experiments at reactors offer the possibility to overcome these limitations with high neutron fluxes. The flux determination is typically based on the activation of two monitors with known cross sections to separate the different flux components. The usually applied cadmium difference method allows a distinction between the thermal and the epithermal part. By a combination of two linear functions representing both monitors the neutron flux components can be determined. However, if more than two monitors are used, the linear system of equations is overdetermined, which allows the identification of a probability distribution. In this proceeding, the feasibility and relevance of this method is demonstrated.
\end{abstract}

\section{Reactor activations}

Reactor activations offer the possibility to determine neutron-induced cross sections of astrophysical interest. The difficulties encountered involving small cross sections or radioactive isotopes can be overcome by high neutron fluxes in the reactor. In particular, experiments with epithermal neutrons providing the resonance integral (RI) allow constraints on the effective cross sections in the astrophysically relevant $\mathrm{keV}$ range. The neutron flux in a reactor can be divided into a thermal, an epithermal component and a component of fast neutrons. The $(\mathrm{n}, \gamma)$ effective cross section decreases rapidly with increasing energy, so that the fast neutrons can be neglected. The thermal component can be suppressed with cadmium because cadmium has a high effective cross section for thermal neutrons [1]. Thus, both the thermal and the epithermal part of the neutron flux can be determined by applying the cadmium difference method.

\section{Analytical determination of the neutron flux}

To determine the neutron flux analytically, the assumption $R_{A}=\sigma_{A}^{t h} \Phi^{t h}+\sigma_{A}^{e p i} \Phi^{e p i}$, with $R_{A}=\frac{N_{A+1}}{N_{A}}$ for an isotope with mass $A$ can be used. $\sigma_{A}^{\text {th }}$ and $\sigma_{A}^{e p i}$ denote the thermal neutron capture cross section and the RI respectively. The thermal flux $\Phi^{\text {th }}$ and epithermal flux $\Phi^{e p i}$ 


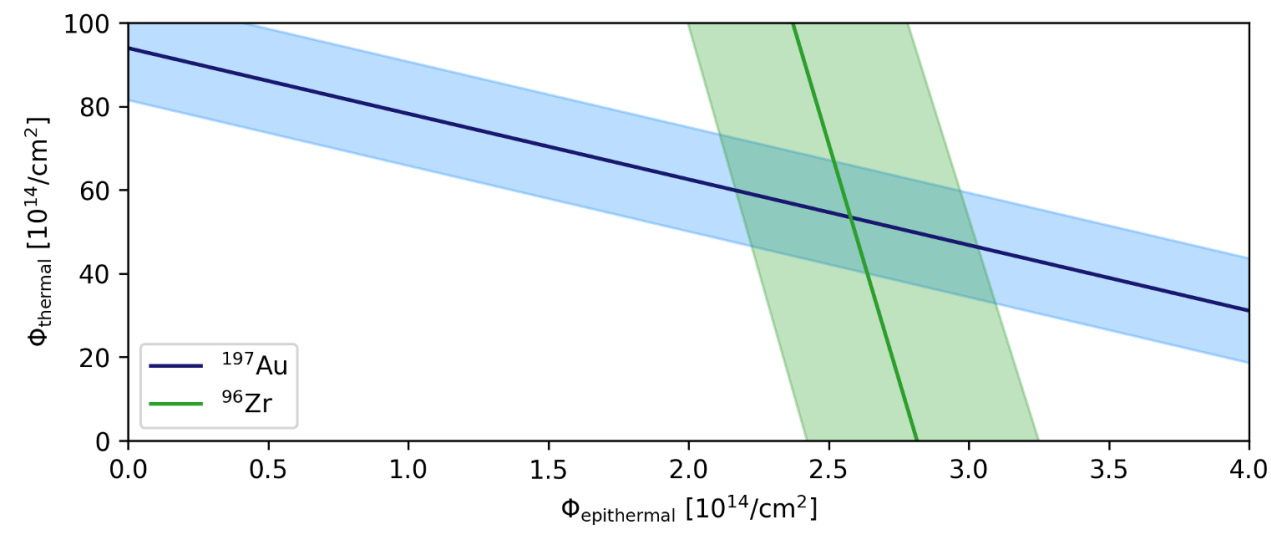

Figure 1. Relation between the thermal neutron flux $\Phi^{\text {th }}$ and the epithermal component $\Phi^{e p i}$ determined by a system of linear equations for two monitors.

components can only be disentangled if at least two monitors with different ratios $\sigma_{A}^{e p i} / \sigma_{A}^{t h}$ and $\sigma_{B}^{e p i} / \sigma_{B}^{\text {th }}$ are used. If only two monitor isotopes are used, the corresponding equations result in a system of linear equations (SLE) and can be analytically solved for $\Phi^{t h}$ and $\Phi^{e p i}$ :

$$
\Phi^{t h}=\frac{\sigma_{B}^{e p i} R_{A}-\sigma_{A}^{e p i} R_{B}}{\sigma_{B}^{e p i} \sigma_{A}^{t h}-\sigma_{A}^{e p i} \sigma_{B}^{t h}}, \Phi^{e p i}=\frac{\sigma_{A}^{t h} R_{B}-\sigma_{B}^{t h} R_{A}}{\sigma_{B}^{e p i} \sigma_{A}^{t h}-\sigma_{A}^{e p i} \sigma_{B}^{t h}}
$$

Figure 1 illustrates the analytical determination by combining two monitors. From the figure it is already obvious that large uncertainties may result from this method.

\section{Probability distribution of the neutron flux}

If more than two monitors are used, the SLE is overdetermined. The idea is now to determine the most likely pair of $\Phi^{\text {th }}$ and $\Phi^{e p i}$. It is therefore necessary to construct the probability distribution for all monitor combinations and each isotope. Thus, the uncertainty for the production ratio $R_{A}$ for each flux pair has to be determined:

$$
\mathrm{d} R_{A}=\sqrt{\left(\Phi^{t h} \mathrm{~d} \sigma_{A}^{t h}\right)^{2}+\left(\Phi^{e p i} \mathrm{~d} \sigma_{A}^{e p i}\right)^{2}}
$$

For the probability distribution follows:

$$
P_{A}^{\Phi}=C_{A} \exp \left(-\frac{\left(R_{A}^{\Phi}-R_{A}^{e x p}\right)^{2}}{2\left(\left(\mathrm{~d} R_{A}^{\Phi}\right)^{2}+\left(\mathrm{d} R_{A}^{e x p}\right)^{2}\right)}\right)
$$

The normalization constant $c_{A}$ has to be determined such that the integral is 1 . The probability distributions for all monitor pairs need to be combined:

$$
P^{\Phi}=c \prod c_{A} P_{A}^{\Phi}
$$

The global normalization constant $c$ has to be determined such that the integral is 1 . By increasing the number of monitors, the flux values can now be determined more precisely. 


\section{Cadmium-encapsulated activation at TRIGA reactor Mainz}

The neutron flux determination can be illustrated by the example of a reactor activation at the TRIGA reactor in Mainz. Four monitors ${ }^{96} \mathrm{Zr},{ }^{96} \mathrm{Zr},{ }^{45} \mathrm{Sc}$ and ${ }^{197} \mathrm{Au}$ covered with a $1 \mathrm{~mm}$ thick cadmium layer were activated for three hours. Figure 2 shows graphically the linear equations for the neutron fluxes in this activation. The probabilities for the respective neutron fluxes are plotted on the split axes.

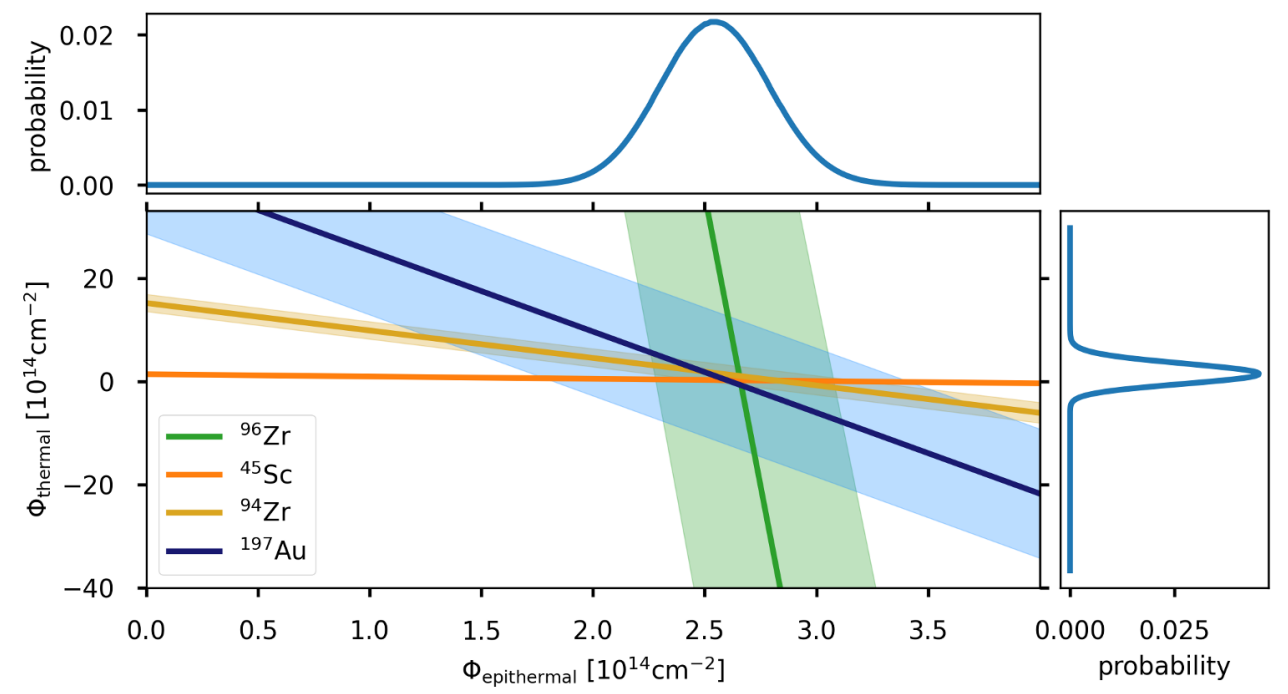

Figure 2. Relation between the thermal $\Phi^{t h}$ the epithermal neutron flux $\Phi^{e p i}$ determined by four monitors. The shared axes show projections of the probability distribution.

Graphically, the fluxes determined by the different methods agree with each other. The data necessary for the calculation are the production rates of the monitors used ${ }^{96} \mathrm{Zr},{ }^{94} \mathrm{Zr}$, ${ }^{45} \mathrm{Sc}$, and ${ }^{197} \mathrm{Au}$, shown in table 1 . The neutron fluxes obtained are shown in table 2 . It lists the values of the neutron fluxes displayed in figure 2. For the analytical determination the monitor pair ${ }^{45} \mathrm{Sc}$ and ${ }^{197} \mathrm{Au}$ was chosen exemplarily. The uncertainty especially for the epithermal part has been reduced drastically.

$$
\begin{array}{cccc}
\mathrm{R}_{95} \mathrm{Zr}^{94} \mathrm{Zr} & \mathrm{R}_{97} \mathrm{Zr}^{96} \mathrm{Zr} & \mathrm{R}^{46} \mathrm{Sc}^{45} \mathrm{Sc} & \mathrm{R}_{198} \mathrm{Au}^{197} \mathrm{Au} \\
\hline(0.73 \pm 0.02) \cdot 10^{-10} & (1.4 \pm 0.002) \cdot 10^{-9} & (3.89 \pm 0.08) \cdot 10^{-9} & (3.99 \pm 0.2) \cdot 10^{-7}
\end{array}
$$

Table 1. Values of the production rates of the four monitors.

\begin{tabular}{lcc} 
& $\Phi^{\text {th }}\left[\mathrm{cm}^{-2}\right]$ & $\Phi^{e p i}\left[\mathrm{~cm}^{-2}\right]$ \\
\hline Analytical determination $\left({ }^{45} \mathrm{Sc}\right.$ and $\left.{ }^{197} \mathrm{Au}\right)$ & $(3.65 \pm 0.59) \cdot 10^{13}$ & $(2.41 \pm 0.05) \cdot 10^{14}$ \\
\hline Most probable neutron flux & $(2.89 \pm 0.51) \cdot 10^{13}$ & $(2.52 \pm 0.04) \cdot 10^{14}$ \\
\hline
\end{tabular}

Table 2. Values for the thermal $\Phi^{\text {th }}$ and the epithermal $\Phi^{e p i}$ neutron flux component determined analyticaly for the monitor pair ${ }^{45} \mathrm{Sc}$ and ${ }^{197} \mathrm{Au}$ and second by using the probability approch.

\section{References}

[1] Frankle, C., Physical Review C Volume 50, p. 2774 (1994) 